\title{
A NON-DESTRUCTIVE SCREENING METHOD FOR PROANTHOCYANIDIN-FREE BARLEY MUTANTS
}

\author{
by \\ HENRIETTE KRISTENSEN ${ }^{1)}$ and STEN AASTRUP ${ }^{2)}$ \\ ${ }^{1)}$ Department of Physiology, Carlsberg Laboratory \\ and \\ ${ }^{2}$ Department of Brewing Chemistry, Carlsberg Research Laboratory, \\ Gamle Carlsberg Vej 10, DK-2500 Copenhagen Valby
}

Keywords: Catechin, leucocyanidin, anthocyanin, vanillin- $\mathrm{HCl}$ colour reaction

Barley grains are embedded in a block of plastic clay with the Carlsberg Seed Fixation System, abraded to expose a small sector of endosperm and surrounding testa. Staining of the proanthocyanidins in the testa is carried out by immersion into a solution of vanillin in $4.5 \mathrm{~N}-\mathrm{HCl}$. Kernels lacking proanthocyanidins are selected, washed and grown to mature plants. Using sodium azide mutagenesis 10 proanthocyanidin-free mutants with anthocyanin pigmentation in the vegetative parts of the plants were obtained among $78,450 \mathrm{M}_{3}$-kernels amounting to a frequency of $0.013 \%$.

\section{INTRODUCTION}

The clear appearance of beer is an important quality factor, since many consumers will rank a hazy beer less satisfactory. The most common forms of haze are precipitates of proteins with proanthocyanidins originating from malt or hops (5). Therefore, proanthocyanidins have to be removed or beer proteins degraded in the brewing process, in order to achieve a colloidally stable beèr. Proanthocyanidin-free barley makes stabilisation of beer superfluous, as has been shown in brewing experiments with the proanthocyanidin-free barley mutant ant 13-13 (10). This has led to extensive breeding programmes for proanthocyanidin-free barley varieties (11).

Proanthocyanidins in the testa of the grains and red anthocyanins in the vegetative part of the plant are formed by the phenylpropanoid and flavonoid pathways. As shown in Figure 1 the common precursor of the two classes of compounds is the (+)-2,3-trans-3,4-cis-leuco- cyanidin (11). A genetic block in the pathway before the branch point, as examplified by mutants in the genes ant 13, ant 21 , ant 17 , ant 22 and ant 18 results in the absence of both anthocyanins and proanthocyanidins. Depending on the location of a biochemical mutation after the branch point either anthocyanin-free plants with normal amounts of catechin and proanthocyanidins are obtained (ant 1-12, 1416), or lines with red anthocyanin pigmentation lacking catechin and proanthocyanidins can be isolated (ant 19). Several hundred mutants, free of anthocyanidins and proanthocyanidins, have been selected by isolating green plants which lacked anthocyanins in $\mathbf{M}_{2}$-populations in the field. From 5 to $25 \%$ of such mutants are found to be proanthocyanidin-free when the kernels are tested with a vanillin- $\mathrm{HCl}$ solution. This test gives a red colour, when catechin or proanthocyanidin are present, and it is carried out on flour from the distal part of the grain, while the 


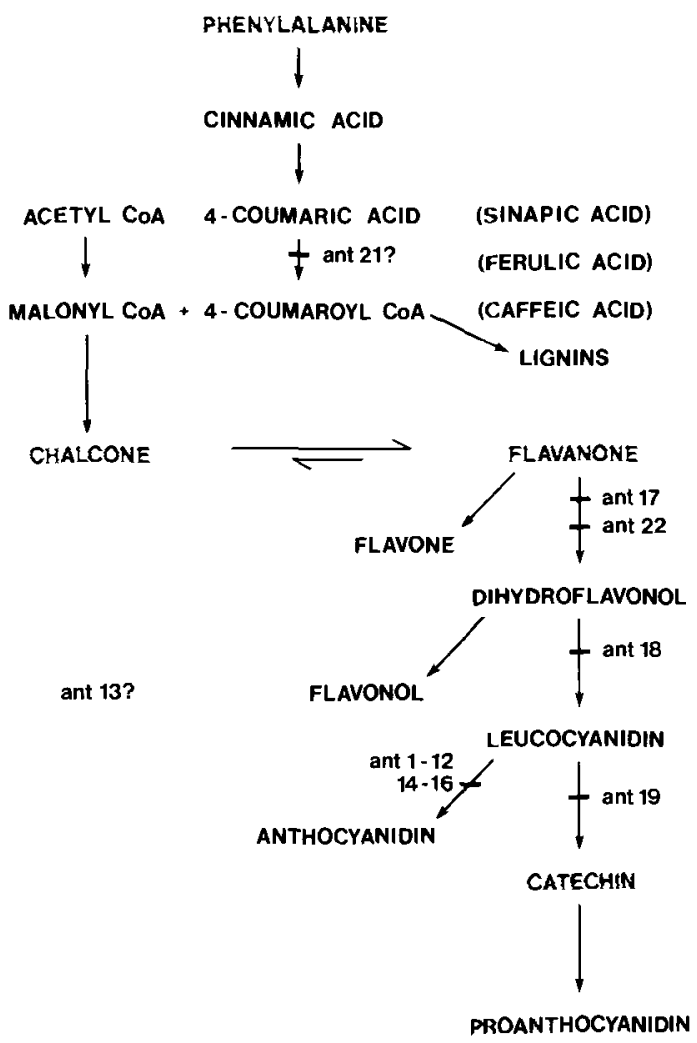

Figure 1. Biosynthesis of proanthocyanidins, anthocyanidins and related compounds. The proposed site of action of different ant genes is indicated (9).

residual part of the grain containing the embryo is kept for germination (9). This selection procedure should not lead to mutants which are blocked in the last step of catechin and proanthocyanidin synthesis i.e. in the portion of the pathway after the branch point, which is specific to the biosynthesis of the latter compounds. Indeed, only very few such mutants have been found so far.

Vanillin staining can be applied on half seeds and thin sections. This permitted the histochemical location of the proanthocyanidins to the testa (4). Using the Carlsberg Seed Fixation System (1) the presence or absence of proanthocyanidins can be determined on sanded surfaces of individual barley or malt grains with the vanillin- $\mathrm{HCl}$ colour reaction, and 400 kernels can be analysed destructively in a period of a few minutes (1), permitting the plant breeder, seed producer, corn dealer and malster to evaluate a portion of proanthocyanidin-free barley for purity in a short time. The aim of the present work was to adapt this procedure to a non-destructive selection of proanthocyanidin-free mutants and recombinants by carrying out the vanillin test on sanded kernel surfaces in such a manner that the embryo remains undamaged. This allows the selected kernels to be germinated and since a large number of grains can be analysed per day, an efficient method for selection of proanthocyanidin-free mutants with the vanillin reaction could be established.

\section{MATERIALS AND METHODS}

\subsection{Grain material}

For the selection of suitable matrices for embedding the kernels and optimal time of staining without damaging the embryo, kernels from the varieties Triumph and Galant (a proanthocyanidin-free mutant in Trumph) were used. For screening of mutagenized material $\mathrm{M}_{3}$-grains harvested on $\mathrm{M}_{2}$-plants are to be used, since the proanthocyanidins are synthesized in the maternal tissue $(3,4,6)$. To demonstrate the practical use of the method a total number of 78,450 $\mathrm{M}_{3}$-kernels of Grit, KM 123 (Research Institute for Cereal Growing and Breeding, Kromeriz, CSSR) and Catrin were analysed. The mutagen treatment of the grains was carried out with a $10^{-3} \mathrm{M}$-sodium azide solution at $\mathrm{pH}$ 3.0 for 2 hrs after presoaking of the grains in distilled water at $+5{ }^{\circ} \mathrm{C}$ for $15 \mathrm{hrs}$ (9). Prior to harvesting the $\mathrm{M}_{2}$-populations were examined for anthocyanidin-free $\mathbf{M}_{2}$-plants, which were removed. This increases the probability that the proanthocyanidin-free mutants found in this material most likely contain anthocyanins.

\subsection{Sample preparation}

The grains are embedded into a block of clay with the Carlsberg Seed Fixation System $(2,7,8)$ consisting of matrix pairs, a press and a disc sander (Figure 2A and B) as follows:

1) Fifty grains are placed into oval-concave depressions of the under-matrix with the aid of the over-matrix containing oval holes (Figure 2C). 


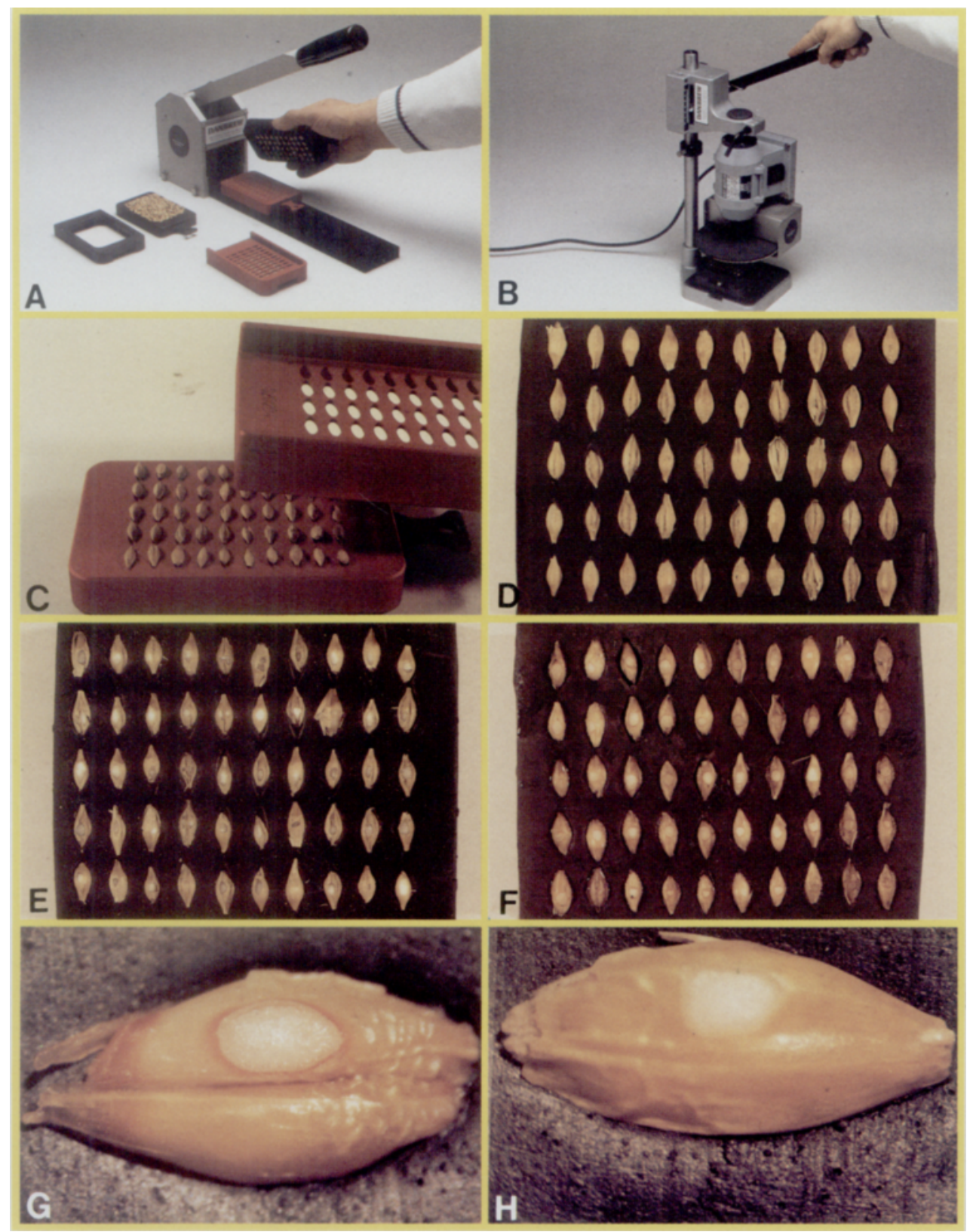

Figure 2. Carlsberg Seed Fixation System.
A: Matrix pairs and press.
B: Disc sander.
C: Over-matrix and under-matrix containing grains.
D: Clay block with grains.
E: Clay block with lightly sanded grains.
F: Clay block with vanillin- $\mathrm{HCl}$ stained grains.
G: Proanthocyanidin-containing grain stained with vanillin- $\mathrm{HCl}$.
$\mathrm{H}$ : Proanthocyanidin-free grain stained with vanillin- $\mathrm{HCl}$. 
2) The grains are fixed in a block of clay, (Filia a/s, Jægersborgvej 64, DK-2800 Lyngby, Denmark) by means of the press (Figure 2D).

3) A small outer part of each grain is removed with the disc sander and this uncovers a sector of the white endosperm (Figure 2E).

\subsection{Staining}

The proanthocyanidins are rendered visible by staining the sanded grains with a freshly prepared solution of $1 \%$ vanillin in $\mathrm{HCl}$. The solution is stable for 3-4 hours. A red colour develops in the testa of proanthocyanidin-containing grains (Figure $2 \mathrm{~F}$ and $\mathrm{G}$ ), while proanthocyanidin-free grains remain colourless (Figure $2 \mathrm{~F}$ and $\mathrm{H}$ ).

\subsection{Selection procedure}

Screening for the proanthocyanidin-free mutants is carried out with the naked eye or a magnifying glass. The optimal position for looking at the plates is from an oblique angle.

Grains lacking a red ring around the endosperm are taken out and instantly placed in a glass of pure water. To rinse out the residual acid the selected grains are placed in running water (e.g. in a small sieve) over night.

The following day, the seeds are sown in vermiculite. A couple of days after germination, they are transfered to soil.

\section{RESULTS AND DISCUSSION}

\subsection{Development of the method}

Grains from the lines Triumph and Galant were used in developing the procedure. Various combinations of acidity of the solution and the times of immersion of the grains were tested to find an acceptable combination of colour intensity and germination ability. The colour development for Triumph was satisfactory after treatment with vanillin in $4.5 \mathrm{~N}-\mathrm{HCl}$ for 20 minutes. Germination of $4 \times 100$ identically treated proanthocyanidin-free Galant grains in vermiculite showed an average germination of $94 \%$ (standard deviation 1.2), whereas untreated grains of this line showed a germination of $97 \%$ (standard deviation 2.3).
Tabel I. Screening for proanthocyanidin-free $\mathrm{M}_{3}$ grains in three barley lines, treated with the mutagen sodium azide.

\begin{tabular}{|c|c|c|c|}
\hline & Grit & KM 123 & Catrin \\
\hline Screened grains & 52200 & 20000 & 6250 \\
\hline $\begin{array}{l}\text { Selected grains } \\
\text { without distinct } \\
\text { red staining }\end{array}$ & 117 & 49 & 10 \\
\hline Germinated grains & 67 & 26 & 8 \\
\hline Survivors & 53 & 21 & 7 \\
\hline $\begin{array}{l}\text { Proanthocyanidin- } \\
\text { free plants }\end{array}$ & 7 & 4 & 2 \\
\hline $\begin{array}{l}\text { Proanthocyanidin- } \\
\text { free plants con- } \\
\text { taining anthocyanin }\end{array}$ & 5 & 3 & 2 \\
\hline
\end{tabular}

\subsection{Application}

The number of proanthocyanidin-free mutants obtained with this method are listed in Table I. In the three lines the frequency of apparently proanthocyanidin-free grains was between $0.16 \%$ and $0.24 \%$. Germination of the selected grains varied from $57 \%$ in Grit, to $53 \%$

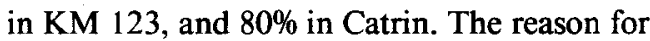
this low germination rate in the progeny of the mutagenized grains as compared to the untreated Galant kernels is possibly due to segregating mutant kernels with decreased viability. The majority of the plants that withered and died after germination were albina mutants.

Whether or not the plant had anthocyanin pigmentation was registered during growth. After harvesting, each plant was checked for proanthocyanidin content with the vanillin test carried out on one fourth of each single grain (9). This test showed that 13 of the selected and surviving plants were proanthocyanidin-free mutants, which is commensurate with $16 \%$. Thus, a portion of the selected grains without red staining testa turned out to contain proanthocyanidin. These escapers might be explained by a weaker colour reaction in some grains upon the vanillin- $\mathrm{HCl}$ treatment, and a tendency to pick all doubtful cases in order to prevent loss of potentially proanthocyanidin-free mutants. Of 
the 13 proanthocyanidin-free mutants 10 plants had anthocyanin pigmentation, corresponding to an induced mutation frequency of $0.013 \%$, calculated on basis of 78,450 screened $\mathrm{M}_{3}$ grains. The mutation frequency for the proanthocyanidin-free mutants lacking anthocyanin can not be calculated, as the main part of the plants without anthocyanin pigmentation was harvested separately.

\section{CONCLUSION}

The vanillin reaction on exposed endosperm surface of grains appropriately embedded in clay, permits the selection of proanthocyanidinfree mutants which are blocked before and after the branch point leading to anthocyanidin and proanthocyanidins, respectively.

It is possible for a person to examine about 1000 grains per hour. The test can be carried out on bulk-harvested grains, and thus eliminates the necessity to harvest plants or ears individually in the field.

\section{ACKNOWLEDGEMENT}

The authors wish to express their appreciation to Professor D. von WetTSTEIN for his encouragement and help in preparation of the manuscript.

\section{REFERENCES}

1. Aastrup, S.: A test for presence or absence of proanthocyanidins in barley and malt. Carlsberg Res. Commun. 50, 37-42 (1985)

2. AASTRUP, S.: Quick, reliable and simple check methods for barley and malt using the Carlsberg Seed Fixation System. J. ASBC, 1986 (in press)

3. AASTRUP, S. \& H. OUTTRUP: Location and characterization of barley flavanoids during grain filling. Proc. Europ. Brewery Conv. Congr., Helsinki, 667-674 (1985)

4. Aastrup, S., H. OUtTRUP \& K. ERdal: Location of the proanthocyanidins in the barley grain. Carlsberg Res. Commun. 49, 105-109 (1984)

5. ERdal, K.: Proanthocyanidin-free barley - Malting and brewing. J. Inst. Brew. 92, 220-224 (1986)

6. FALK, D. E.: Genetic studies with proanthocyanidin-free barley. Barley Genet. Newslett. 15, 27-30 (1985)

7. Heltved, F., S. Aastrup. O. Jensen, G. Gibbons \& L. MUNCK: Preparation of seeds for mass screening. Carlsberg Res. Commun. 47, 291-296 (1982)

8. JENSEN, S. AA. \& S. AASTRUP: Determination of malt modification. Cerevisia 10, 113-123 (1985)

9. WetTStein, D. von, B. JeNDE-Strid, B. AhrenstLARSEN \& K. ERDAL: Proanthocyanidin-free barley prevents the formation of beer haze. MBAA Tech. Quart. 17, 16-23 (1980)

10. Wettstein. D. von, B. Jende-Strid, B. AhrenstLARSEN \& J. A. SøRENSEN: Biochemical mutant in barley renders chemical stabilization of beer superfluous. Carlsberg Res. Commun. 42, 341-351 (1977)

11. Wettstein, D. von, R. A. Nilan, B. AhrenstLarSen, K. ERdal, J. IngVersen, B. Jende-Strid, K. Nyegaard Kristiansen. J. Larsen, H. OUtTRUP \& S. E. UlLRICH: Proanthocyanidin-free barley for brewing: Progress in breeding for high yield and research tool in polyphenol chemistry. MBAA Tech. Quart. 22, 41-52 (1985)

Accepted by E. LuND 